\title{
Epidemiology of Patient Monitoring Alarms in the Neonatal Intensive Care Unit
}

\author{
Taibo Li, MEng ${ }^{1}$, Minoru Matsushima, PhD ${ }^{1,2}$, Wendy Timpson, MD $^{3}$, Susan Young, RNC- \\ NIC, SM$^{3}$, David Miedema, BS ${ }^{3}$, Munish Gupta, MD $^{3}$, and Thomas Heldt, PhD ${ }^{1}$ \\ ${ }^{1}$ Institute for Medical Engineering \& Science, Massachusetts Institute of Technology, Cambridge, \\ MA, United States \\ ${ }^{2}$ Nihon Kohden Innovation Center, Cambridge, MA, United States \\ ${ }^{3}$ Department of Neonatology, Beth Israel Deaconess Medical Center, Boston, MA, United States
}

\section{Abstract}

Objective-To characterize the rate of monitoring alarms by alarm priority, signal type and developmental age in a Level-IIIB Neonatal Intensive Care Unit (NICU) population.

\begin{abstract}
Study Design—Retrospective analysis of 2294687 alarm messages from Philips monitors in a convenience sample of 917 NICU patients, covering 12001 patient-days. We stratified alarm rates by alarm priority, signal type, postmenstrual age (PMA), and birth weight (BW) and reviewed and adjudicated over 21000 critical alarms.
\end{abstract}

\begin{abstract}
Results-Of all alarms, $3.6 \%$ were critical alarms, $55.0 \%$ were advisory alarms, and $41.4 \%$ were device alerts. Over $60 \%$ of alarms related to oxygenation monitoring. The average alarm rate $( \pm$ SEM) was 177.1 \pm 4.9 [median: 135.9; IQR: 89.2-213.3] alarms/patient-day; the medians varied
\end{abstract}

Users may view, print, copy, and download text and data-mine the content in such documents, for the purposes of academic research, subject always to the full Conditions of use: http://www.nature.com/authors/editorial_policies/license.html\#terms

Address correspondence to: Thomas Heldt, PhD, Institute for Medical Engineering \& Science, Massachusetts Institute of Technology, Building E25, Room 324, 77 Massachusetts Avenue, Cambridge, MA, 02139, United States, thomas@ mit.edu, Phone: 617-324-5005.

Contributors' Statements

Mr. Li led the data analysis, interpreted the results, helped draft the initial manuscript, critically reviewed and revised the manuscript, and approved the final manuscript as submitted.

Dr. Matsushima contributed to the data analysis, interpreted the results, helped draft and critically review and revise the initial manuscript, and approved the final manuscript as submitted.

Dr. Timpson and Ms. Young provided clinical perspective on the study, interpreted the results, critically reviewed and revised the initial manuscript, and approved the final manuscript as submitted.

Mr. Miedema supported the clinical data extraction and data collection, critically reviewed the initial manuscript, and approved the final manuscript as submitted.

Dr. Gupta contributed to and supported the data collection, provided clinical perspective on the study, interpreted the results, critically reviewed and revised the initial manuscript, and approved the final manuscript as submitted.

Dr. Heldt conceptualized, designed, and supervised all aspects of the study, including data collection, data analysis, overall study progress, and drafting and revising of the manuscript. He critically reviewed the manuscript and approved the final manuscript as submitted.

Conflict of Interest

Dr. Heldt has received research funding from the National Institutes of Health, Nihon Kohden Corporation, Maxim Integrated, and Philips Healthcare. Dr. Matsushima remained a salaried employee of Nihon Kohden Corporation while contributing to this study as a Visiting Scientist at MIT's Institute for Medical Engineering \& Science. Mr. Li received partial internship support from Nihon Kohden Innovation Center. Ms. Young, Mr. Miedema, Dr. Timpson, and Dr. Gupta declare no potential conflict of interest.

Supplementary Information accompanies the paper on the Journal of Perinatology website (http://www.nature.com/jp). 
significantly with PMA and BW $(\mathrm{p}<0.001)$ in $\mathrm{U}$-shaped patterns, with higher rates at lower and higher PMA and BW. Based on waveform reviews, over $99 \%$ of critical arrhythmia alarms were deemed technically false.

Conclusions-The alarm burden in this NICU population is very significant; the average alarm rate significantly underrepresents alarm rates at low and high PMA and BW. Virtually all critical arrhythmia alarms were artifactual.

\section{Introduction}

Providing effective life support to the critically ill relies heavily on an array of bedside monitoring devices to track the physiological state of each patient and to alert care providers - through visual and audible alarm messages - to significant changes in key physiological parameters. In the preterm population, for example, oxygenation monitoring is critically important to draw attention to dangerously low or high oxygenation levels. Monitoring devices also alert care providers to technical problems with the measurements or the devices themselves. These alarming features are indispensable in modern technology-driven critical care. The proliferation of bedside monitoring, however, has resulted in high rates of monitoring alarms, most of which are thought to be either technically false or clinically irrelevant ${ }^{1,2,3,4}$, leading to desensitization of care providers ('alarm fatigue') ${ }^{2,4,5}$ and the potential to miss truly life-threatening deterioration, thus putting patients at serious risk.

In the context of neonatal intensive care, several recent studies have reported and analyzed sizeable volumes of alarm data to understand alarm patterns and to suggest possible strategies to reduce the overall alarm burden. ${ }^{6,7,8,9,10}$ However, few studies have comprehensively quantified the overall NICU alarm load by developmental age of the infant. The care and associated monitoring strategies of the preterm neonate are primarily determined by the infant's pathophysiology and illness severity. However, the care and monitoring strategies typically change with gestational and postmenstrual age, and the alarm types and alarm burden are therefore also likely to change over the course of an infant's NICU stay. A comprehensive characterization and stratification of NICU alarm patterns may help identify opportunities to reduce the overall alarm burden and increase patient safety.

In the present study, we provide such systematic classification of the alarm burden for a tertiary-care NICU population. We report on the distribution of patient monitoring alarms by signal type and alarm priority, and stratify the alarm rates by postmenstrual age and birth weight. Additionally, we manually reviewed a subset of all critical alarms to assess the degree to which the issued alarms are technically true or technically false.

\section{METHODS}

\section{Clinical Setting, Study Design and Study Sample}

We performed a cross-sectional retrospective data analysis on a convenience sample of infants admitted between February 2008 and June 2013 to the Level-IIIB NICU at Boston's Beth Israel Deaconess Medical Center (BIDMC), a Harvard Medical School teaching hospital. The study was approved by the Institutional Review Boards at BIDMC and MIT. 
The requirement for individual patient consent was waived because the study did not impact clinical care and all protected health information was deidentified.

The BIDMC NICU consists of 24 separate patient rooms, each accommodating up to two patients. The patient rooms are arranged along the outside margin of a large U-shaped corridor. Staff work areas and six central monitoring stations are located along the inner margin, with each central station capable of covering up to six patient rooms. The cardiorespiratory status of all infants can thereby be observed both directly at the bedside, as well as at a central monitor. For less acutely ill infants, the nurse-to-infant ratio is 1:3 irrespective of nursing shift. For infants of higher acuity, the ratio is increased to 1:2 or 1:1 depending on the complexity of care required. Furthermore, infants of higher acuity are admitted to patient rooms at the bottom of the $\mathrm{U}$ where the concentration of staff is typically higher and response time is optimized.

Approximately 5000 infants are delivered annually at BIDMC, which has an active high-risk obstetrical service. Of the 1300 annual NICU admissions, the vast majority are inborn and approximately half are admitted for 24 hours or less. Infants with complex cardiac or surgical conditions are transferred to Boston Children's Hospital.

\section{Patient Monitoring}

At BIDMC, each NICU bed is equipped with a dedicated patient monitor (IntelliVue MP-90, Philips Healthcare, Andover, MA) that acquires, digitizes, and displays physiological waveform data; computes and displays physiological trend data; and evaluates monitoring alarm conditions and issues alarm notifications. While the degree of monitoring varies with gestational age (GA), postmenstrual age (PMA), and patient condition and acuity, commonly acquired physiological waveforms include the electrocardiogram (ECG) and pulse plethysmogram (PPG). In subsets of infants, continuous positive airway pressure (CPAP) is acquired, and arterial blood pressure (ABP) may be recorded via an indwelling arterial catheter. Computed physiological trend data include heart rate (HR), arterial oxygen saturation $\left(\mathrm{SpO}_{2}\right)$, respiratory rate (Resp), and arterial blood pressure metrics (systolic, mean, and diastolic). Ventilators, infusion pumps and bed alarms are not interfaced to the Philips monitor. Alarms from these and other stand-alone bedside devices were therefore not studied herein.

Throughout the study, the ECG was acquired using the Philips IntelliVue ECG module, and the PPG and $\mathrm{SpO}_{2}$ signals were based on Nellcor Oximax MAX-NS and SC-PR probes directly interfaced to the IntelliVue monitors. The PPG sensor was generally repositioned once per nursing shift, and the ECG electrodes were repositioned or replaced on an as-need basis.

\section{Multiparameter Data Archiving}

The physiological waveforms, trend data, and alarm notifications from all patient monitors are transmitted over the dedicated hospital monitoring network to the central stations (M3155 IntelliVue Information Center, Philips Healthcare) and to a hospital database server for temporary storage. Archiving of the patient monitoring data occurred as part of the Multiparameter Intelligent Monitoring in Intensive Care II (MIMIC-II) project. ${ }^{11}$ Briefly, a 
dedicated archiving agent was developed and deployed in collaboration with Philips Healthcare and was designed to copy the transmitted data packages in real-time from the hospital's monitoring network to a local fileserver to permanently archive the multiparameter physiologic monitoring data and alarm messages for research use. Because of bandwidth limitations at the time, we were only able to copy the data packages, and therefore archive patient monitoring data, from half of the 48 NICU beds. Physiological waveforms were transmitted at $125 \mathrm{samples} / \mathrm{sec}$ and trend data at one sample/sec. Timestamped alarm messages were transmitted at the time of alarm issuance at the bedside. The archived records were retrieved regularly from the archiving agents, de-identified, and converted from a Philips proprietary format to an open-source data format. ${ }^{12}$

\section{Alarm Types, Settings, Escalation, and Notifications}

The Philips patient monitoring system classifies alarms into three priority levels: INOPs, yellow (advisory), and red (critical). ${ }^{13}$ INOPs are technical alarms that are issued in response to measurement or data display problems and may indicate that alarm conditions cannot be evaluated reliably. ${ }^{13}$ Yellow alarms are low-priority physiological alarms that call attention to arrhythmias or alarm-limit (threshold) violations that are not considered acutely life threatening. Red alarms are high-priority alarms and indicate the potential presence of a life-threatening condition or critical device failure. In our population, red alarms include critical desaturation, critical tachycardia, critical bradycardia, asystole, ventricular tachycardia/ventricular fibrillation (VTach/VFib), and non-pulsatile pressure signals with amplitudes less than $10 \mathrm{mmHg}$ ("pressure disconnects"). The full unit-wide alarm settings by PMA, including alarm thresholds, delays and averaging times, are provided in the Supplementary Information (Table S1).

By unit-wide default, the respiratory rate, apnea, and yellow arrhythmia alarms were disabled. However, care providers were able to activate respiratory rate alarms at the bedside and alter the alarm-limit settings for all threshold-based alarms except for critical desaturation alarms. Red arrhythmia alarms (asystole and VTach/VFib) also could not be disabled. Alarms issued at the bedside were relayed to the central monitoring stations and to other patient rooms when care providers chose to utilize the patient overview option that displays alarm messages issued by one patient's monitor on another patient's monitor. No further alarm escalation to individual care providers was in place at the time of our data capture.

The archived Philips alarm notifications consist of the time stamp when the alarm was issued at the bedside and an alarm message that reports the alarm condition and associated alarm priority (red, yellow, or INOP). For alarm-limit violations, the alarm messages also contain the value of the prevailing alarm limit (threshold) and the measured deviation of the signal from the alarm threshold at the time of alarm notification. The archived alarm notifications do not contain any information about alarm silencing or alarm suspension. During clinical care, the alarm-pause feature is usually activated prior to routine handling of an infant. The NICU staff typically silence yellow alarms prior to or as they respond to the alarm. Additionally, yellow alarms are non-latching in our NICU and will reset without caregiver intervention if the alarm condition resolves. Red alarms prompt direct patient 
evaluation. Red alarms are also latching, which means they require caregiver response to be silenced.

\section{Alarm Adjudications}

Since waveform signal artifacts can mimic true physiological events we set out to determine whether issued red alarms were technically true or false. To that end, we reviewed the ECG and PPG waveforms preceding asystole and VTach/VFib alarms, and the PPG waveform and $\mathrm{SpO}_{2}$ trend data in a subset of the critical desaturation alarms. Asystole alarms were judged to be true if the ECG waveform was free of artifacts in the 20 -second interval preceding the time of asystole alarm and if there was no evidence of cardiac activity for four seconds or longer in the ECG or any of the other monitoring waveforms (principally the PPG waveform). Likewise, VTach/VFib alarms were judged to be true if the ECG waveform was free of artifacts in the 20-second interval preceding the time of VFib/VTach alarm, the cardiac activity could credibly pass as a ventricular rhythm, and information from other monitoring signals (again principally the PPG waveform) did not contradict the alarm condition. A critical desaturation event was deemed correct if a pulsatile PPG waveform was discernable, and the $\mathrm{SpO}_{2}$ signal was free of artifact and met the indicated alarm condition within the 20 seconds preceding the critical desaturation alarm. If an asystole, VTach/VFib, or critical desaturation alarm was not explicitly determined to be true, we considered it to be technically false.

\section{Clinical Data}

For each infant admitted to the NICU over the duration of the study period, we extracted from the electronic medical records the medical record number (MRN), first and last name, gestational age (GA), gender, date of birth, birth weight (BW), and NICU admit and discharge dates and times.

\section{Patient Matching and Exclusion Criteria}

Since the MIMIC-II data acquisition protocol did not store bed labels, the archived monitoring data could only be associated with certainty with a particular infant if the clinical staff manually entered the infant's name and/or MRN into the monitoring system. Since such entry of infant information into the patient monitor was done inconsistently, we were only able to match a subset of all archived patient monitoring records to associated clinical information. We excluded from our analysis all infants for whom archived records could not be matched to clinical information with certainty. We also excluded infants that were admitted to the NICU for less than 24 hours and infants for whom less than sixty minutes of total patient monitoring data were archived. We did not impose any exclusion on the basis of signal type or alarm priority.

\section{Statistical Analysis}

To determine the overall number and fraction of alarms by alarm priority (red, yellow, INOP) and signal type (ECG, PPG, ABP, CPAP), we extracted from each alarm notification the alarm condition, alarm priority, and alarm limits breached (for threshold alarms). To determine the per-patient alarm rate, we additionally extracted for each infant the total 
duration for which at least one monitoring signal was available. We compute the per-patient alarm rate as the total number of alarms in a patient's record divided by the duration over which monitoring data were available. We normalize each infant's per-patient alarm rate to a 24-hour monitoring period and report the resultant alarm rate as alarms/patient-day.

Given that recording durations can vary significantly for different signal types, we also computed the per-patient "active-signal" alarm rates as the total number of alarms of a particular signal type (ECG, PPG, ABP, CPAP) divided by the total duration for which the particular signal was available (actively monitored) in a given patient. We report the activesignal alarm rate as alarms/24 hours of signal availability.

From the per-patient alarm rates, we compute the average alarm rates and associated standard errors of the mean (SEM) by averaging across all per-patient alarm rates in our patient pool. We also report the median and interquartile ranges (IQR) for some alarm rates. We stratify alarm rates by alarm priority (red, yellow, INOP), signal type, PMA, and BW. We use the Anderson-Darling approach to test for normality of the alarm rate distributions for each PMA and BW category. Alarm rates stratified by PMA or BW are compared statistically using the Kruskal-Wallis test. A p-value of 0.05 was used to indicate statistical significance. Finally, to assess the extent to which care providers adjust alarm settings from the unit-wide default thresholds for $\mathrm{HR}$ and $\mathrm{SpO}_{2}$ alarms, we compute the fraction of $\mathrm{HR}$ and $\mathrm{SpO}_{2}$ threshold alarms for which the breached thresholds conformed to the unit-wide default.

\section{RESULTS}

Of the 7119 infants admitted to the BIDMC NICU between February 2008 and June 2013, 99\% were inborn and 3462 had a length-of-stay (LOS) exceeding 24 hours. Of those with LOS exceeding 24 hours, $72 \%$ were preterm and $17 \%$ were Very Low Birth Weight (VLBW) or Extremely Low Birth Weight (ELBW). From the MIMIC-II database, we retrieved multiparameter patient monitoring data of 60 minutes or longer for 917 of these infants. Table 1 summarizes the demographic information of our study population and the overall NICU population that met the date range and LOS inclusion requirement. While no attempt was made to generate a (statistically) representative sample of the NICU population, clinical characteristics of our study sample correlate with the characteristics of the overall NICU population across GA, BW, and LOS (see Supplementary Information, Figures S1S3).

The 917 infants accounted for a total of 27009.4 NICU days (74.0 patient-years), of which we captured 12001.1 days (32.9 patient-years) in the MIMIC-II database (44.4\% matching). Over the captured period, we recorded a total of 2294687 monitoring alarms, 3.6\% of which were red alarms, $55.0 \%$ yellow alarms, and $41.4 \%$ INOPs (Figure 1). $\mathrm{PPG} / \mathrm{SpO}_{2}$ related (red, yellow, and INOP) alarms accounted for $60.3 \%$ of all monitoring alarms, followed by ECG-related alarms (28.1\%), Resp (5.4\%), ABP (4.7\%), and CPAP (1.2\%). Of the INOP alarms, the most common notifications related to a non-pulsatile or noisy $\mathrm{SpO}_{2}$ signal (34.6\%); ECG leads off, noisy ECG signal or inability to analyze the ECG (29.0\%); as well as noisy or non-pulsatile $\mathrm{ABP}$ and display issues of the $\mathrm{ABP}$ waveform (10.5\%). 
The average alarm rate was 177.1 \pm 4.9 [median: 135.9; IQR: 89.2-213.3] alarms/patient-day, or - on average - one monitoring alarm every eight minutes per infant. With $89.8 \pm 2.9$ [median: 60.1; IQR: 36.6-109.3] alarms/patient-day, the PPG signal accounted for the majority of alarms issued per patient-day, followed by the ECG with $63.8 \pm 2.4$ [median: 47.8; IQR: 28.9-76.8] alarms/patient-day (Table 2). When normalized by the time over which each signal was available, the ABP and CPAP-related alarm rates dominate. These signals generated a sizeable number of INOP alarms, but were only recorded in a small fraction of patients and for a comparatively short period of time, which amplified their active-signal alarm rates.

When we stratified the alarm rates by PMA, we observed a U-shaped relationship in average alarm rate with PMA (Figure 2) and major birth-weight categories (Figure 3). Arterial blood pressure INOP alarms (non-pulsatile signal, noisy signal, and monitor display size issues) and $\mathrm{SpO}_{2} \mathrm{High} / \mathrm{Low}$ alarms contribute disproportionately to the average alarm rate at low PMAs. Increases in the ECG (noisy signal, leads off, 'cannot analyze ECG') and PPG (low perfusion, non-pulsatile signal, noisy signal) INOP alarms, as well as an increase in $\mathrm{SpO}_{2}$ Low alarms, are responsible for the rise in alarm rates above 34 weeks PMA. The distributions of alarm rates by PMA and birth-weight categories failed the test for normality, but statistically significant differences $(\mathrm{p}<0.001)$ between alarm rates were observed, likewise with U-shaped relationships, when the median alarm rates were stratified by PMA and major birth-weight categories (Supplementary Information, Figures S4 and S5).

During the adjudication process, we reviewed the ECG and PPG waveforms associated with 16784 red arrhythmia alarms (asystole or VTach/VFib) and 4273 red desaturation alarms, for a total review of 21057 critical alarms. We adjudicated 100\% (14 216/14 216) of asystole alarms, 26.9\% of the VTach/VFib (2 568/8 883) alarms, and 18.9\% (4 273/22 204) of the critical desaturation alarms. In this sample, $99.9 \%$ (14 195/14 216) of all asystole alarms, essentially 100\% (2 567/2 568) of VTach/VFib alarms, and 35.9\% (1 535/4 273) of the critical desaturation alarms were deemed technically false according to the criteria described in the Methods section, most commonly due to saturation artifacts or loss of signal.

The threshold settings for $96.9 \%$ of all HR Low alarms, $94.4 \%$ of HR High alarms, $89.2 \%$ of critical bradycardia alarms, and $96.0 \%$ of critical tachycardia alarms conformed to the unitwide default settings. Likewise, the threshold settings for $80.5 \%$ of $\mathrm{SpO}_{2}$ Low alarms, $78.5 \%$ of $\mathrm{SpO}_{2} \mathrm{High}$ alarms, and $99.6 \%$ of critical desaturation alarms conformed to the unit-wide default settings. Of those threshold-crossing alarms that did not conform to the unit-wide default settings, a high fraction occurred at PMAs at which the unit-wide default thresholds are supposed to change for $\mathrm{SpO}_{2}$ High alarms (PMA of 32 and 35 weeks), $\mathrm{SpO}_{2}$ Low alarms (PMA of 35 weeks), and HR Low and critical bradycardia alarms (PMA of 34 weeks).

\section{Discussion}

The proliferation of bedside monitoring alarms remains a challenge in modern critical care and poses a clear and tangible risk to patient safety. ${ }^{2}$ In the United States, the Joint 
Commission has mandated that hospitals establish alarm safety as an organizational priority and take a principled approach to alarm system management. ${ }^{14}$ To help identify opportunities to reduce the alarm burden, we set out to comprehensively characterize the rates of patient monitoring alarms in a sizeable Level-IIIB NICU population. This study complements recent work on neonatal patient monitoring alarm burden and management, $6,7,8,9,10$ by stratifying alarm rates by PMA and BW, in addition to alarm priority and signal type.

The average alarm rate reported here is significantly lower than those reported recently by Ketko et al. ${ }^{9}$ and by McClure et al. ${ }^{10}$ in Level-IV NICU populations. Our average alarm rate is also lower than that reported by Vergales et al. $^{6}$ in a VLBW population. These differences may be related to the respective patient populations and acuity, and to the number and types of signals monitored. For example, our NICU does not provide care for infants with complex cardiac or surgical conditions. Also, respiratory rate and apnea alarms are disabled by unitwide default because of exceedingly high rates of nuisance alarms associated with these signals. Nevertheless, our results demonstrate that the aggregate alarm burden in this LevelIIIB NICU population is very high, resulting - on average - in one alarm per patient every eight minutes. Additionally, the alarm rates reported here represent a low estimate of the true bedside alarm burden in our NICU as our analysis focused exclusively on alarms issued by the Philips patient monitor; it did not account for alarms issued by warmers, incubators, ventilators, infusion pumps, or other stand-alone bedside devices.

A key finding of our study is that the average alarm rate significantly underestimates the alarm rates at low and high PMAs and BWs. ABP INOP alarms and $\mathrm{SpO}_{2}$ High/Low alarms contribute significantly to the rise in alarm rates at low PMA. In our population, invasive $\mathrm{ABP}$ monitoring is used predominantly in very preterm infants and is associated with a very high active-signal alarm rate (Table 2) consisting mostly of "low-amplitude" INOP alarms. At higher PMAs, an increase in PPG- and ECG-related INOP alarms contributes to a modest increase in the alarm rate. This increase may be related to motion artifacts in both PPG and ECG signals due to increased activity in older infants. Additionally, according to our NICU guidelines, our $\mathrm{SpO}_{2}$ Low alarm limit is increased from $87 \%$ to $92 \%$ at 35 weeks PMA, which contributes to an increased rate of desaturation alarms above 35 weeks PMA.

Schondelmeyer et al. ${ }^{15}$ recently reported that INOP alarms constitute $33 \%$ of the overall alarm burden in a pediatric hospital population. In our study population INOP alarms also contribute to a very significant degree (41.4\%). Since the vast majority of INOP alarms are $\mathrm{PPG} / \mathrm{SpO}_{2}$ or ECG related, this finding suggests that improvements in the reliable acquisition of the PPG and ECG signals continue to have very significant potential to reduce the overall alarm burden. Despite routine repositioning of the PPG sensor once per nursing shift, the $\mathrm{PPG} / \mathrm{SpO}_{2}$ alarm rate still dominated the alarm burden in our NICU. Reductions in NICU PPG/SpO 2 alarm rates have been reported for some sensor technologies, ${ }^{16,17,18}$ though most studies were conducted in comparatively small numbers of infants or over relatively short observation periods. Daily ECG electrode changes have been shown to reduce nuisance alarms in adult ICU populations. ${ }^{19,20}$ To our knowledge no such study has been conducted in a NICU population, and the effect size of such changes on NICU alarm rate is therefore currently unknown. 
Yellow alarms account for the majority of all alarm notifications in our study population and consist exclusively of alarm-limit violations. Various prior studies have investigated how changes in averaging time ${ }^{21}$, alarm delay ${ }^{21,22}$, or alarm limits ${ }^{9,21}$ affect the incidence of threshold-crossing alarms, particularly $\mathrm{SpO}_{2}$ alarms. Longer averaging times, longer alarm delays, and wider alarm limits all result in a decreased number of threshold-crossing events. 10,23 In this study, $\mathrm{SpO}_{2} \mathrm{High} / \mathrm{Low}$ alarms account for the vast majority of all yellow (advisory) alarms. The alarm delay and averaging time for $\mathrm{SpO}_{2} \mathrm{High} / \mathrm{Low}$ alarms in our unit are $10 \mathrm{~s}$ and $20 \mathrm{~s}$, respectively. The latter is the maximal value allowed by the manufacturer and therefore results in the maximal attenuation of transient $\mathrm{SpO}_{2}$ excursions that is possible with our patient monitors. Our alarm delay is shorter than that recently advocated by McClure et al., who report that $55 \%$ of the $\mathrm{SpO}_{2} \mathrm{High} / \mathrm{Low}$ threshold crossing events in their cohort last less than 15 seconds. ${ }^{10}$ Increasing our alarm delay to $15 \mathrm{~s}$ might therefore further reduce the incidence of $\mathrm{SpO}_{2} \mathrm{High} / \mathrm{Low}$ alarms. To reduce the incidence of threshold-crossing events, clinicians can also adjust and therefore personalize the alarm thresholds for individual infants. In our population, however, the vast majority of the breached alarm limits conform to the unit-wide default settings. Changes to the averaging time, alarm delay, and alarm thresholds need to be balanced against the potential for missing clinically relevant events. Since our database did not contain information about which threshold alarms were clinically relevant or actionable, stratification of alarms according to clinical relevance was not possible.

Only 3.6\% of all alarms were of the red (critical) alarm priority. While the incidence of red alarms is comparatively low in our population, the associated false alarm rates are very high. Since care providers must respond expeditiously to potentially life-threatening events, improvements in the specificity of critical alarms would also liberate care provider time, reduce distraction and thereby improve patient safety. Our alarm adjudications confirm that true asystole and VTach/VFib alarms are exceedingly rare in the neonatal population. The vast majority of true asystole alarms resolved spontaneously shortly after the alarm condition was met and did not require provider intervention. Only three asystole events lasted longer than ten seconds, and all three occurred in infants on comfort measures only close to the end of life.

In reviewing the physiological waveforms during the alarm adjudication process, we had the benefit of drawing from information across different waveform signals. Basing alarm conditions on information from multiple physiological signal streams, rather than a single signal, can significantly reduce the high rate of technically false critical alarms ${ }^{24,25}$ and should become part of standard patient monitoring algorithms as the overwhelming majority of critical arrhythmia alarms were simply technically false.

The current study is limited by its single-center nature, and the results must therefore be interpreted in the context of the local unit-specific policies, patient population, and alarm settings (see Supplementary Information). Additionally, the archiving infrastructure limited data collection to half of the NICU beds, and matching of waveform records to clinical records relied on provider entry of patient information in the monitoring system. It is unlikely that these limitations led to significant systematic selection bias, as infants were assigned beds largely based on bed availability rather than infant characteristics. It is 
possible that identifiers were entered into monitors more reliably for infants with certain characteristics such as longer LOS. Overall, however, the population of infants that were matched to waveform data were similar to the overall NICU population (Table 1;

Supplementary Information).

A further limitation is the lack of alarm annotations by clinical relevance or action taken. Ideally, each alarm should be labeled as clinically relevant/actionable, clinically helpful, clinically irrelevant, or care-provider induced. ${ }^{1}$ Such alarm annotations would need to be captured at the time the alarm is issued and action is taken at the bedside. They would be particularly helpful in devising rational approaches to reducing the high incidence of threshold-crossing alarms.

The work presented here is the first detailed accounting of NICU bedside monitoring alarms by PMA and BW in a sizeable cohort. Our analysis demonstrates that the rate of bedside monitoring alarms in this Level-IIIB NICU population varies significantly with PMA and $\mathrm{BW}$, and is largely driven by non-critical threshold-crossing and INOP alarms. Identification of the factors that drive alarm frequency lays the groundwork for future work to reduce unnecessary alarm burden.

\section{Supplementary Material}

Refer to Web version on PubMed Central for supplementary material.

\section{Acknowledgments}

The authors wish to thank Mr. Jeffrey Smith, Clinical Engineering, BIDMC, for his help and support over the course of this study. Additionally, the authors thank Mr. Nirmal Balachundhar, Mr. Rémi Dekimpe, Ms. Tiffany Ho, and Ms. Nalini Singh for their help with the adjudication of bedside monitoring alarms. The data collection and archiving was supported in part through grants R01 EB001659 and R01 GM104987 from the National Institute of Biomedical Imaging and Bioengineering and National Institute of General Medical Sciences, respectively, of the United States National Institutes of Health. The data analysis was supported in part by Nihon Kohden Corporation and Nihon Kohden Innovation Center, and in part by a Massachusetts Institute of Technology (MIT) Peter J. Eloranta Summer Undergraduate Research Fellowship and the MIT-Wertheimer Undergraduate Research and Innovation Scholar Fund. Preliminary analyses of this work were presented as a poster at the 2016 Pediatric Academic Societies Annual Meeting in Baltimore, MD.

\section{References}

1. Tsien CL, Fackler JC. Poor prognosis for existing monitors in the intensive care unit. Crit Care Med. 1997; 25(4):614-619. [PubMed: 9142025]

2. Johnson KR, Hagadorn JI, Sink DW. Alarm Safety and Alarm Fatigue. Clin Perinatol. 2017; 44(3): 713-728. [PubMed: 28802348]

3. Paine CW, Goel VV, Ely E, Stave CD, Stemler S, Zander M, et al. Systematic Review of Physiologic Monitor Alarm Characteristics and Pragmatic Interventions to Reduce Alarm Frequency. J Hosp Med. 2016; 11(2):136-144. [PubMed: 26663904]

4. Association for the Advancement of Medical Instrumentation. Clinical Alarms - 2011 summit. Arlington, VA: Association for the Advancement of Medical Instrumentation; 2011.

5. Cvach M. Monitor alarm fatigue: an integrative review. Biomed Instrum Technol. 2012; 46(4):268277. [PubMed: 22839984]

6. Vergales BD, Paget-Brown AO, Lee H, Guin LE, Smoot TJ, Rusin CG, et al. Accurate automated apnea analysis in preterm infants. Am J Perinatol. 2014; 31(2):157-162. [PubMed: 23592319] 
7. Joshi R, van Pul C, Atallah L, Feijs L, Van Huffel S, Andriessen P. Pattern discovery in critical alarms originating from neonates under intensive care. Physiol Meas. 2016; 37(4):564-579. [PubMed: 27027383]

8. van Pul C, HPVDM, JJVDB, Mohns T, Andriessen P. Safe patient monitoring is challenging but still feasible in a neonatal intensive care unit with single family rooms. Acta Paediatr. 2015; 104(6):e247-254. [PubMed: 25619759]

9. Ketko AK, Martin CM, Nemshak MA, Niedner M, Vartanian RJ. Balancing the Tension Between Hyperoxia Prevention and Alarm Fatigue in the NICU. Pediatrics. 2015; 136(2):e496-504. [PubMed: 26148949]

10. McClure C, Jang SY, Fairchild K. Alarms, oxygen saturations, and $\mathrm{SpO} 2$ averaging time in the NICU. J Neonatal Perinatal Med. 2016; 9(4):357-362. [PubMed: 27834782]

11. Saeed M, Villarroel M, Reisner AT, Clifford G, Lehman LW, Moody G, et al. Multiparameter Intelligent Monitoring in Intensive Care II: a public-access intensive care unit database. Crit Care Med. 2011; 39(5):952-960. [PubMed: 21283005]

12. The WFDB Software Package. 2015. [cited 2015 January 4]Available from: http://physionet.org/ physiotools/wfdb.shtml

13. Philips. IntelliVue Patient Monitor MP 20/30, MP 40/50, MP 60/70/90 - Instructions for Use. Germany: 2005.

14. The Joint Commmission. 2017 National Patient Safety Goals. 2017. [cited 2017 May 25]Available from: https://www.jointcommission.org/hap_2017_npsgs/

15. Schondelmeyer AC, Bonafide CP, Goel VV, Blake N, Cvach M, Sucharew H, et al. The frequency of physiologic monitor alarms in a children's hospital. J Hosp Med. 2016; 11(11):796-798. [PubMed: 27233467]

16. Ahlborn V, Bohnhorst B, Peter CS, Poets CF. False alarms in very low birthweight infants: comparison between three intensive care monitoring systems. Acta Paediatr. 2000; 89(5):571-576. [PubMed: 10852195]

17. Sahni R, Gupta A, Ohira-Kist K, Rosen TS. Motion resistant pulse oximetry in neonates. Arch Dis Child Fetal Neonatal Ed. 2003; 88(6):F505-508. [PubMed: 14602699]

18. Stefanescu BM, O’Shea TM, Haury F, Carlo WA, Slaughter JC. Improved Filtering of Pulse Oximeter Monitoring Alarms in the Neonatal ICU: Bedside Significance. Respir Care. 2016; 61(1):85-89. [PubMed: 26508772]

19. Cvach MM, Biggs M, Rothwell KJ, Charles-Hudson C. Daily electrode change and effect on cardiac monitor alarms: an evidence-based practice approach. J Nurs Care Qual. 2013; 28(3):265271. [PubMed: 23187092]

20. Sendelbach S, Wahl S, Anthony A, Shotts P. Stop the Noise: A Quality Improvement Project to Decrease Electrocardiographic Nuisance Alarms. Crit Care Nurse. 2015; 35(4):15-22. quiz 11p following 22. [PubMed: 26232798]

21. Rheineck-Leyssius AT, Kalkman CJ. Influence of pulse oximeter settings on the frequency of alarms and detection of hypoxemia: Theoretical effects of artifact rejection, alarm delay, averaging, median filtering or a lower setting of the alarm limit. J Clin Monit Comput. 1998; 14(3):151-156. [PubMed: 9676861]

22. Gorges M, Markewitz BA, Westenskow DR. Improving alarm performance in the medical intensive care unit using delays and clinical context. Anesth Analg. 2009; 108(5):1546-1552. [PubMed: 19372334]

23. Vagedes J, Poets CF, Dietz K. Averaging time, desaturation level, duration and extent. Arch Dis Child Fetal Neonatal Ed. 2013; 98(3):F265-266. [PubMed: 22960097]

24. Zong W, Moody GB, Mark RG. Reduction of false arterial blood pressure alarms using signal quality assessment and relationships between the electrocardiogram and arterial blood pressure. Med Biol Eng Comput. 2004; 42(5):698-706. [PubMed: 15503972]

25. Aboukhalil A, Nielsen L, Saeed M, Mark RG, Clifford GD. Reducing false alarm rates for critical arrhythmias using the arterial blood pressure waveform. J Biomed Inform. 2008; 41(3):442-451. [PubMed: 18440873] 


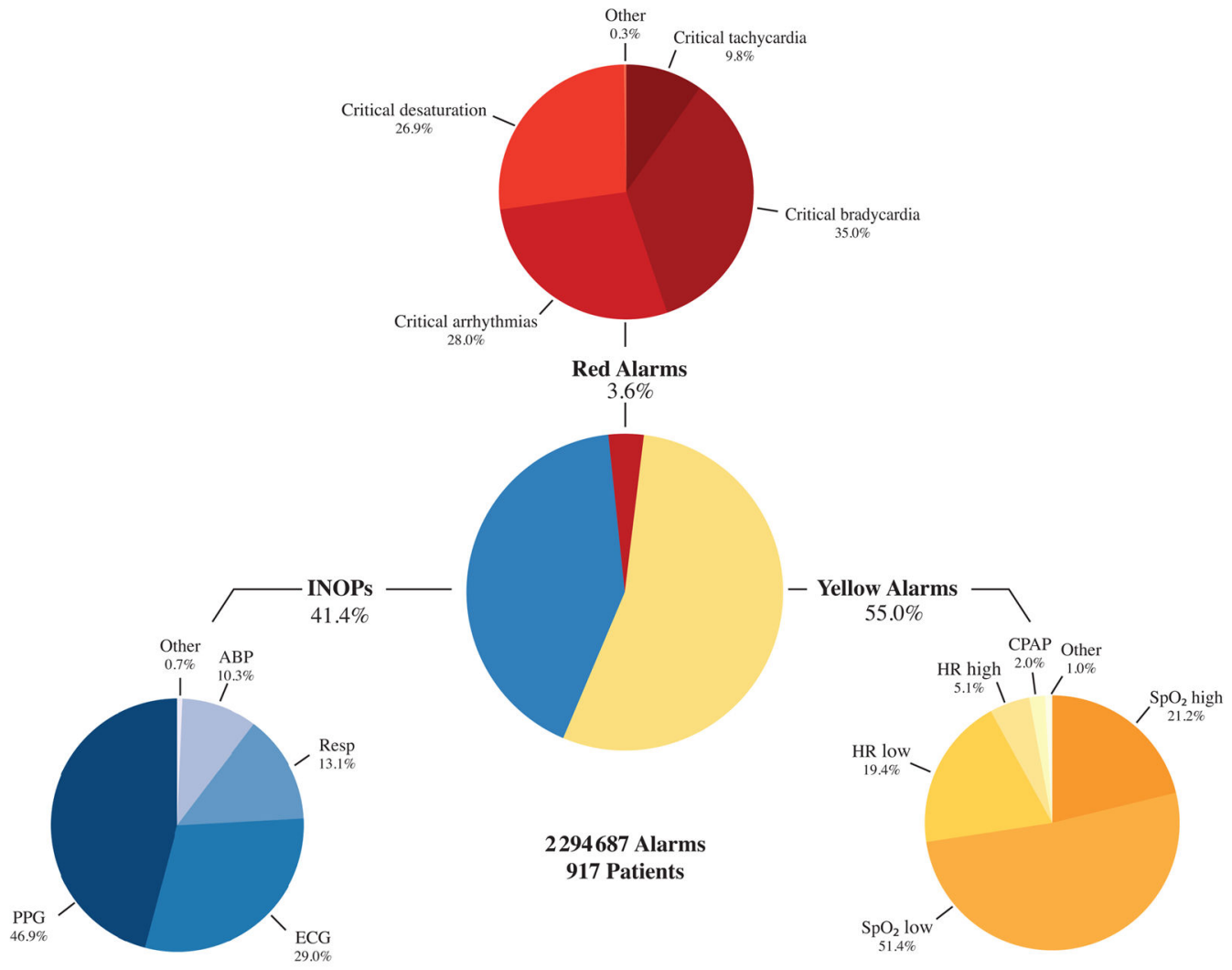

Figure 1.

Distribution of NICU patient monitoring alarms by alarm priority: red (critical) alarms, yellow (advisory) alarms, and INOPs (device alerts). $\mathrm{SpO}_{2}$ : blood oxygenation; CPAP: continuous positive airway pressure; HR: heart rate; ABP: arterial blood pressure; Resp: respiratory rate; ECG: electrocardiogram; PPG: pulse plethysmogram. 


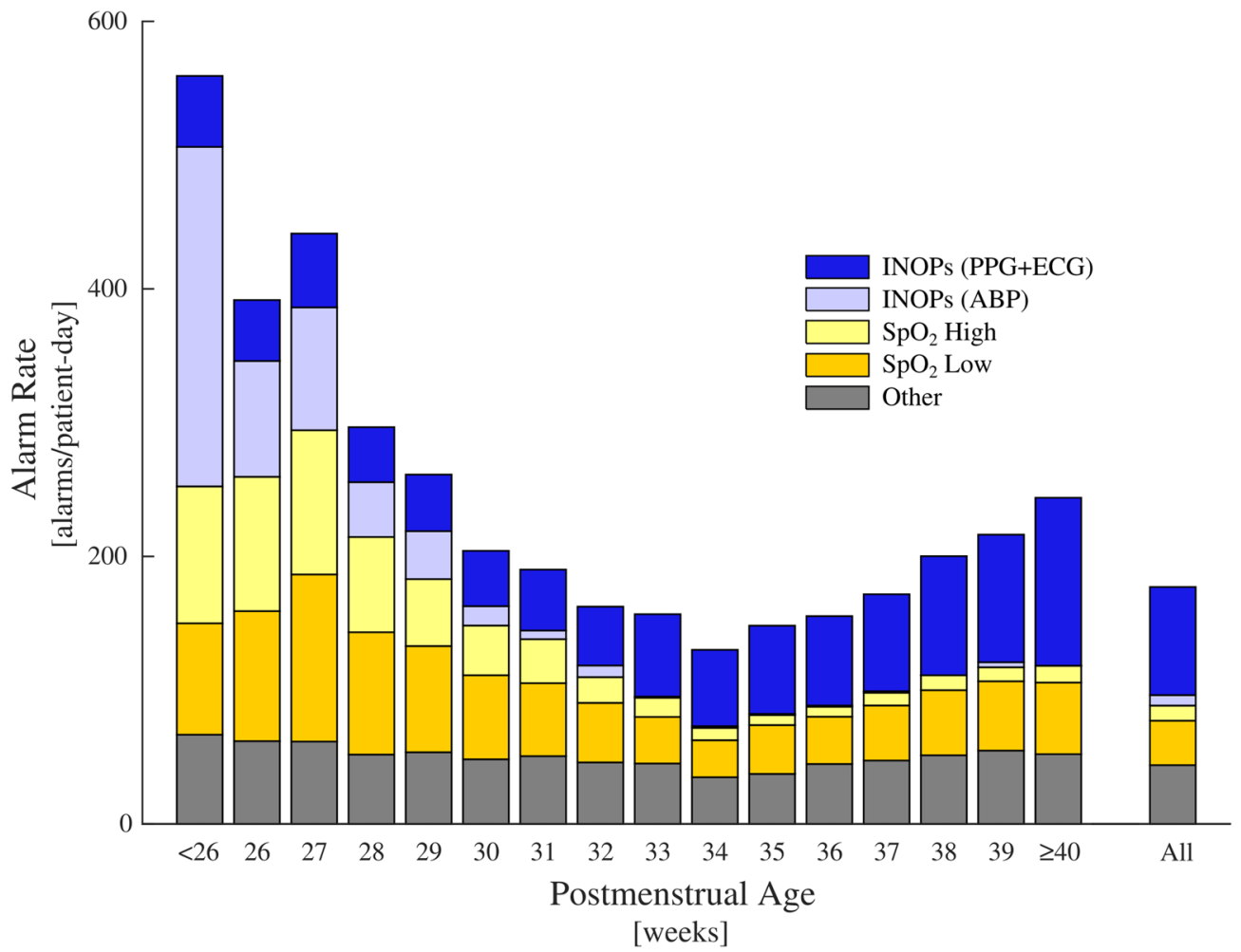

Figure 2.

Average alarm rates, stratified by postmenstrual age, broken down into constituent categories to reveal major alarm rate trends. All: average alarm rate across all 917 patients. 


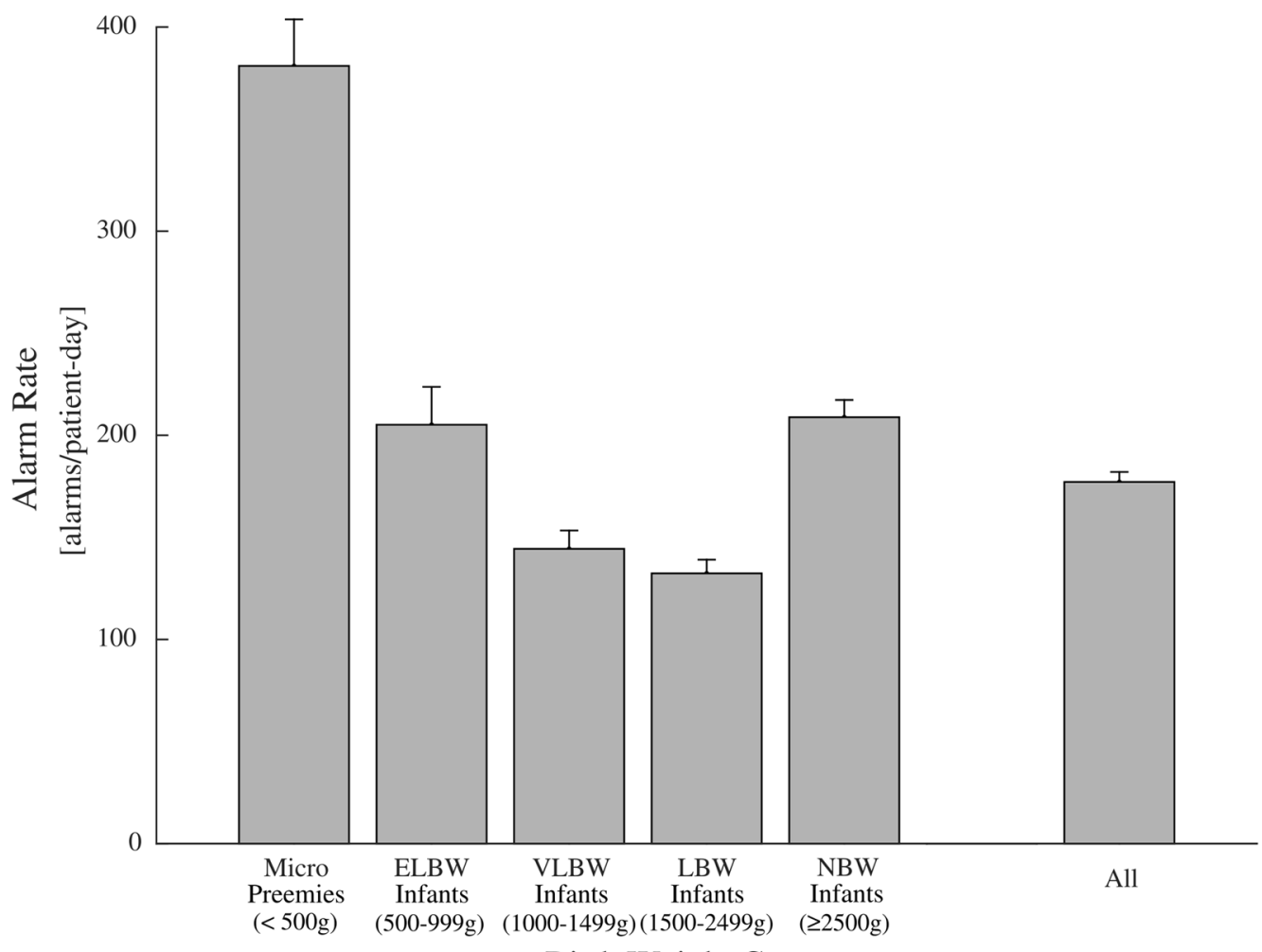

Birth Weight Category

Figure 3.

Average alarm rates $( \pm$ SEM) for micro preemies, ELBW infants, VLBW infants, LBW infants, infants of normal birth weight (NBW), and the entire study population (All). 


\section{Table 1}

Demographics of study population and overall NICU population meeting inclusion/exclusion criteria. Gestational age, birth weight, length-of-stay, and data coverage are given as median and inter-quartile range (in parentheses). Data coverage is the fraction of total NICU time for which we were able to retrieve bedside monitoring data.

\begin{tabular}{lcc}
\hline & Study Population & NICU Population \\
\hline Number of patients & 917 & 3462 \\
\hline Female [\%] & 45.9 & 45.6 \\
\hline Gestational age [weeks] & $34.0(31.1-35.6)$ & $34.6(32.5-37.0)$ \\
\hline Birth weight [g] & $2050(1450-2650)$ & $2270(1729-2$ 915) \\
\hline Length of stay [days] & $17.4(7.7-40.3)$ & $11.4(3.8-26.8)$ \\
\hline Data coverage [\%] & $50.2(24.8-81.4)$ & N/A \\
\hline
\end{tabular}




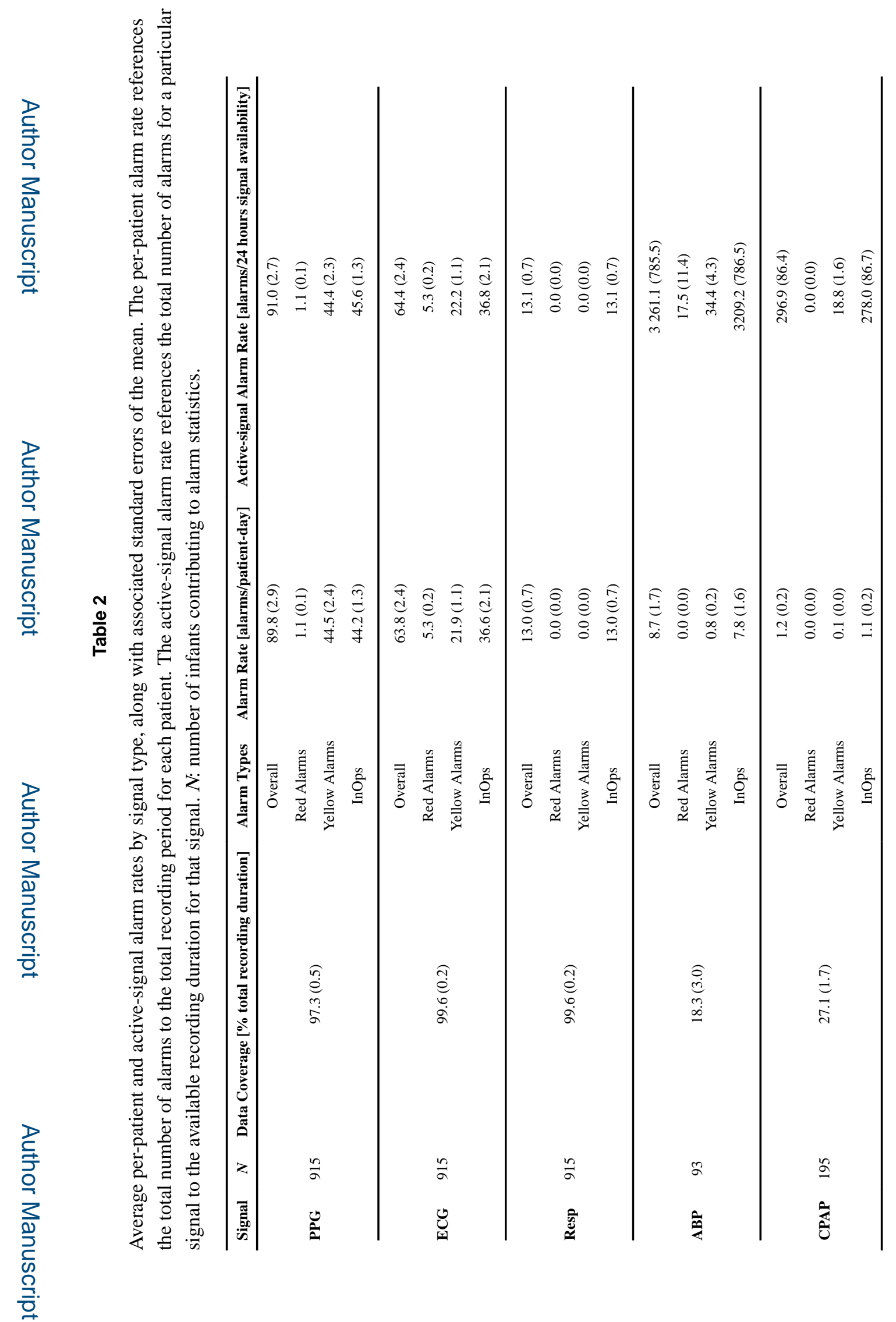

J Perinatol. Author manuscript; available in PMC 2018 November 08. 